\title{
Periodic homogenization of a pseudo-parabolic equation via a spatial-temporal decomposition
}

\author{
Arthur. J. Vromans and Fons van de Ven and Adrian Muntean
}

\begin{abstract}
Pseudo-parabolic equations have been used to model unsaturated fluid flow in porous media. In this paper it is shown how a pseudo-parabolic equation can be upscaled when using a spatio-temporal decomposition employed in the Peszyńska-Showalter-Yi paper [8]. The spatial-temporal decomposition transforms the pseudo-parabolic equation into a system containing an elliptic partial differential equation and a temporal ordinary differential equation. To strengthen our argument, the pseudo-parabolic equation has been given advection/convection/drift terms. The upscaling is done with the technique of periodic homogenization via two-scale convergence. The well-posedness of the extended pseudo-parabolic equation is shown as well. Moreover, we argue that under certain conditions, a non-local-in-time term arises from the elimination of an unknown.
\end{abstract}

Acknowledgements We acknowledge the Netherlands Organisation of Scientific Research (NWO) for the MPE grant 657.000.004 and we acknowledge the NWO Cluster Nonlinear Dynamics in Natural Systems (NDNS+) for funding a research stay of AJV at Karlstads Universitet.

\section{Introduction}

Groundwater recharge and pollution prediction for aquifers need models for describing unsaturated fluid flow in porous media. Pseudo-parabolic equations were found to be adequate models, see eqn. 25 in [3]. In [8] a spatial-temporal decomposition

A.J. Vromans and A.A.F. van de Ven

Centre for Analysis, Scientific computing and Applications (CASA), Eindhoven University of Technology, Den Dolech 2, 5612AZ, Eindhoven, The Netherlands, e-mail: a.j.vromans@tue.nl; a.a.f.v.d.ven@tue.nl

A. Muntean

Department of Mathematics and Computer Science, Karlstad University, Universitetsgatan 2, 651

88, Karlstad, Sweden, e-mail: adrian.muntean@kau.se 
of a pseudo-parabolic system was introduced. It was shown that this decomposition made upscaling of this system rather straightforward in several classical situations such as vanishing time-delay and double-porosity systems. In [8] a toy pseudoparabolic model was derived from a balance equation describing flow through a partially saturated porous medium. In our framework, a convective term that was dropped in [8], is retained in order to show that this term yields no additional problems for upscaling with the spatial-temporal decomposition. We want to convey the message that this decomposition can be applied not only to the physical system in [8] but also to other physical systems with pseudo-parabolic equations, such as the concrete corrosion reaction model introduced in [9]. Both these pseudo-parabolic systems are physical systems on a spatial micro scale with an intrinsic microscopic periodicity of size $\varepsilon \ll 1$. Similar intrinsic microscopic periodic behaviors are found in highly active research fields using composite structures or nano-structures.

In this paper, we use this spatial-temporal decomposition to upscale our pseudoparabolic equation by using the concept of periodic homogenization via two-scale convergence, which leads to a homogenized system that retains the spatial-temporal decomposition. We start in Section 2 with formulating our pseudo-parabolic system $\left(\mathbf{Q}^{\varepsilon}\right)$, the decomposition system $\left(\mathbf{P}^{\varepsilon}\right)$ and stating our assumptions. In Section 3 , an existence and uniqueness result for weak solutions to our problem $\left(\mathbf{P}^{\varepsilon}\right)$ is derived. In Section 4, we apply the idea of two-scale convergence to a weak version of problem $\left(\mathbf{P}^{\varepsilon}\right)$, denoted $\left(\mathbf{P}_{w}^{\varepsilon}\right)$, that contains the microscopic information at the $\varepsilon$-level. Furthermore in this section, an upscaled system $\left(\mathbf{P}_{w}^{0}\right)$ of the weak system $\left(\mathbf{P}_{w}^{\varepsilon}\right)$ is derived in the limit $\varepsilon \downarrow 0$, and, under certain conditions, an upscaled strong system $\left(\mathbf{P}_{s}^{0}\right)$ is obtained after eliminating several variables. This upscaled strong system contains a non-local-in-time term, but the system has lost the partial differential equation framework as a consequence. Contrary, the upscaled weak system $\left(\mathbf{P}_{w}^{0}\right)$ keeps the partial differential equation framework due to the spatial-temporal decomposition.

\section{Basic system and assumptions}

Our pseudo-parabolic system $\left(\mathbf{Q}^{\varepsilon}\right)$ consists of a family of $N$ partial differential equations for the variable vector $\mathbf{U}^{\varepsilon}(t, \mathbf{x}, \mathbf{x} / \varepsilon)=\left(U_{1}^{\varepsilon}, \ldots, U_{\alpha}^{\varepsilon}, \ldots, U_{N}^{\varepsilon}\right)$ with $t>0$ and $\mathbf{x}=\left(x_{1}, \ldots, x_{i}, \ldots, x_{d}\right) \in \Omega \subset \mathbf{R}^{d}$. For $\varepsilon \in\left(0, \varepsilon_{0}\right)$ with $\varepsilon_{0}>0$, system $\left(\mathbf{Q}^{\varepsilon}\right)$ is formulated as

$$
\left(\mathbf{Q}^{\varepsilon}\right) \begin{cases}\mathrm{M}^{\varepsilon} \mathrm{G}^{-1} \partial_{t} \mathbf{U}^{\varepsilon}-\nabla \cdot\left(\left(\mathrm{E}^{\varepsilon} \cdot \nabla+\mathrm{D}^{\varepsilon}\right) \mathrm{G}^{-1}\left(\partial_{t} \mathbf{U}^{\varepsilon}+\mathrm{L} \mathbf{U}^{\varepsilon}\right)\right) & \\ \quad=\mathbf{H}^{\varepsilon}+\left(\mathrm{K}^{\varepsilon}-\mathrm{M}^{\varepsilon} \mathrm{G}^{-1} \mathrm{~L}\right) \mathbf{U}^{\varepsilon}+\mathrm{J}^{\varepsilon} \cdot \nabla \mathbf{U}^{\varepsilon} & \text { on } \mathbf{R}_{+} \times \Omega, \\ \mathbf{U}^{\varepsilon}=\mathbf{U}_{*} & \text { on }\{0\} \times \Omega, \\ \partial_{t} \mathbf{U}^{\varepsilon}+\mathrm{L} \mathbf{U}^{\varepsilon}=\mathbf{0} & \text { on } \mathbf{R}_{+} \times \partial \Omega .\end{cases}
$$


The vectors $\mathbf{V}^{\varepsilon}$ and $\mathbf{U}^{\varepsilon}$ are both functions of the time coordinate $t$, the global or macro position coordinate $\mathbf{x}$, and also periodic functions of the micro (or nano) coordinate $\mathbf{y} \in Y$, where $\mathbf{y}=\mathbf{x} / \varepsilon$, where the size of the micro domain $Y$ is $\mathscr{O}(\varepsilon)$ of the size of the macro domain $\Omega$.

Our dimensionless decomposition system $\left(\mathbf{P}^{\varepsilon}\right)$ consists of a family of $N$ partial differential equations (PDEs) and a family of $N$ ordinary differential equations (ODEs) for the two variable vectors $\mathbf{V}^{\varepsilon}(t, \mathbf{x}, \mathbf{x} / \varepsilon)=\left(V_{1}^{\varepsilon}, \ldots, V_{\alpha}^{\varepsilon}, \ldots, V_{N}^{\varepsilon}\right)$ and $\mathbf{U}^{\varepsilon}(t, \mathbf{x}, \mathbf{x} / \varepsilon)$. For $\varepsilon \in\left(0, \varepsilon_{0}\right)$ with $\varepsilon_{0}>0$, it is formulated as

$$
\begin{cases}\mathrm{M}^{\varepsilon} \mathbf{V}^{\varepsilon}-\nabla \cdot\left(\mathrm{E}^{\varepsilon} \cdot \nabla \mathbf{V}^{\varepsilon}+\mathrm{D}^{\varepsilon} \mathbf{V}^{\varepsilon}\right)=\mathbf{H}^{\varepsilon}+\mathrm{K}^{\varepsilon} \mathbf{U}^{\varepsilon}+\mathrm{J}^{\varepsilon} \cdot \nabla \mathbf{U}^{\varepsilon} & \text { on } \mathbf{R}_{+} \times \Omega, \\ \partial_{t} \mathbf{U}^{\varepsilon}+\mathrm{L} \mathbf{U}^{\varepsilon}=\mathrm{G} \mathbf{V}^{\varepsilon} & \text { on } \mathbf{R}_{+} \times \Omega, \\ \mathbf{U}^{\varepsilon}=\mathbf{U}_{*} & \text { on }\{0\} \times \Omega, \\ \mathbf{V}^{\varepsilon}=\mathbf{0} & \text { on } \mathbf{R}_{+} \times \partial \Omega .\end{cases}
$$

Above, the $\varepsilon$-dependent notation $c^{\varepsilon}(t, \mathbf{x})=c(t, \mathbf{x}, \mathbf{x} / \varepsilon)$ is used for the $\varepsilon$-independent 1-,2- and 3-tensors of assumption (A1).

(A1) For all $\alpha, \beta \in\{1, \ldots, N\}$ and for all $i, j \in\{1, \ldots, d\}$, we have

$$
\begin{aligned}
\mathrm{M}_{\alpha \beta}, \mathrm{E}_{i j}, \mathrm{D}_{i \alpha \beta}, \mathbf{H}_{\alpha}, \mathrm{K}_{\alpha \beta}, \mathrm{J}_{i \alpha \beta} & \in L^{\infty}\left(\mathbf{R}_{+} \times \Omega ; C_{\#}(Y)\right), \\
\mathrm{L}_{\alpha \beta}, \mathrm{G}_{\alpha \beta} & \in L^{\infty}\left(\mathbf{R}_{+} ; W^{1, \infty}(\Omega)\right), \\
\mathbf{U}_{*} & \in C^{1}(\Omega)^{N},
\end{aligned}
$$

with $\mathrm{G}$ invertible.

(A2) Let the tensors $\mathrm{M}^{\varepsilon}$ and $\mathrm{E}^{\varepsilon}$ be in diagonal form ${ }^{1}$ with elements $m_{\alpha}^{\varepsilon}>0$ and $e_{i}^{\varepsilon}>0$, respectively, satisfying $1 / m_{\alpha}^{\varepsilon}, 1 / e_{i}^{\varepsilon} \in L^{\infty}\left(\mathbf{R}_{+} \times \Omega ; C_{\#}(Y)\right)$.

(A3) The inequality

$$
\left\|\mathrm{D}_{i \beta \alpha}^{\varepsilon}\right\|_{L^{\infty}\left(\mathbf{R}_{+} \times \Omega^{\varepsilon} ; C_{\#}(Y)\right)}^{2}<\frac{4}{d N^{2}\left\|1 / m_{\alpha}^{\varepsilon}\right\|_{L^{\infty}\left(\mathbf{R}_{+} \times \Omega^{\varepsilon} ; C_{\#}(Y)\right)}\left\|1 / e_{i}^{\varepsilon}\right\|_{L^{\infty}\left(\mathbf{R}_{+} \times \Omega^{\varepsilon} ; C_{\#}(Y)\right)}}
$$

holds for all $\alpha, \beta \in\{1, \ldots, N\}$, for all $i \in\{1, \ldots, n\}$, and for all $\varepsilon \in\left(0, \varepsilon_{0}\right)$.

Remark, inequality (2) implies that automatically (2) holds for the $Y$-averaged functions $\overline{\mathrm{D}_{i \beta \alpha}^{\varepsilon}}, \overline{\mathrm{M}_{\beta \alpha}^{\varepsilon}}$, and $\overline{\mathrm{E}_{i j}^{\varepsilon}}$ in $L^{\infty}\left(\mathbf{R}_{+} \times \Omega\right)$, using $|Y| \bar{f}(t, \mathbf{x})=\int_{Y} f(t, \mathbf{x}, \mathbf{y}) \mathrm{d} \mathbf{y}$.

\section{Existence and uniqueness of weak solutions to $\left(\mathbf{P}_{w}^{\varepsilon}\right)$}

In this section, we show the existence and uniqueness of a weak solution $(\mathbf{U}, \mathbf{V})$ to $\left(\mathbf{P}^{\varepsilon}\right)$. We define a weak solution to $\left(\mathbf{P}^{\varepsilon}\right)$ for $\varepsilon \in\left(0, \varepsilon_{0}\right)$ and $T \in \mathbf{R}_{+}$as a pair of

${ }^{1}$ Due to the Theorem of Jacobi about quadratic forms (cf. [4]) in combination with the coercivity of both $\mathrm{M}^{\varepsilon}$ and $\mathrm{E}^{\varepsilon}$, we are allowed to assume diagonal forms of $\mathrm{M}^{\varepsilon}$ and $\mathrm{E}^{\varepsilon}$ as the orthogonal transformations, necessary to put their quadratic forms in diagonal form, modify the domain $\Omega^{\varepsilon}$ and the coefficients of $\mathrm{D}^{\varepsilon}, \mathbf{H}^{\varepsilon}, \mathrm{K}^{\varepsilon}$ and $\mathrm{J}^{\varepsilon}$ without changing their regularity. 
sequences $\left(\mathbf{U}^{\varepsilon}, \mathbf{V}^{\varepsilon}\right) \in H^{1}((0, T) \times \Omega)^{N} \times L^{\infty}\left((0, T), H_{0}^{1}(\Omega)\right)^{N}$ satisfying

$$
\left(\mathbf{P}_{w}^{\varepsilon}\right)\left\{\begin{array}{l}
\int_{\Omega} \phi^{\top}\left[\mathrm{M}^{\varepsilon} \mathbf{V}^{\varepsilon}-\mathbf{H}^{\varepsilon}-\mathrm{K}^{\varepsilon} \mathbf{U}^{\varepsilon}-J^{\varepsilon} \cdot \nabla \mathbf{U}^{\varepsilon}\right]+(\nabla \phi)^{\top} \cdot\left(\mathrm{E}^{\varepsilon} \cdot \nabla \mathbf{V}^{\varepsilon}+\mathrm{D}^{\varepsilon} \mathbf{V}^{\varepsilon}\right) \mathrm{d} \mathbf{x}=0, \\
\int_{\Omega} \psi^{\top}\left[\partial_{t} \mathbf{U}^{\varepsilon}+\mathrm{L}^{\varepsilon} \mathbf{U}^{\varepsilon}-\mathrm{G}^{\varepsilon} \mathbf{V}^{\varepsilon}\right] \mathrm{d} \mathbf{x}=0, \\
\mathbf{U}^{\varepsilon}(0, \mathbf{x})=\mathbf{U}_{*}(\mathbf{x}) \text { for all } \mathbf{x} \in \Omega,
\end{array}\right.
$$

for a.e. $t \in(0, T)$, for all test-functions $\phi \in H_{0}^{1}(\Omega)^{N}$ and $\psi \in L^{2}(\Omega)^{N}$.

The existence and uniqueness can only hold when the first equation of $\left(\mathbf{P}_{w}^{\varepsilon}\right)$ satisfies all the conditions of Lax-Milgram. The next lemma provides the coercivity condition, while the continuity condition is trivially satisfied.

Lemma 1. Assume assumptions (A1) - (A3) hold, then there exist positive constants $\tilde{m}_{\alpha}, \tilde{e}_{i}, \tilde{H}, \tilde{K}_{\alpha}, \tilde{J}_{i \alpha}$ for $\alpha \in\{1, \ldots, N\}$ and $i \in\{1, \ldots, d\}$ such that the following a-priori estimate holds for a.e. $t \in(0, T)$.

$$
\begin{aligned}
\sum_{\alpha=1}^{N} \tilde{m}_{\alpha}\left\|\mathbf{V}_{\alpha}^{\varepsilon}\right\|_{L^{2}(\Omega)}^{2} & +\sum_{i=1}^{d} \sum_{\alpha=1}^{N} \tilde{e}_{i}\left\|\partial_{x_{i}} \mathbf{V}_{\alpha}^{\varepsilon}\right\|_{L^{2}(\Omega)}^{2} \\
& \leq \tilde{H}+\sum_{\alpha=1}^{N} \tilde{K}_{\alpha}\left\|\mathbf{U}_{\alpha}^{\varepsilon}\right\|_{L^{2}(\Omega)}^{2}+\sum_{i=1}^{d} \sum_{\alpha=1}^{N} \tilde{i}_{i \alpha}\left\|\partial_{x_{i}} \mathbf{U}_{\alpha}^{\varepsilon}\right\|_{L^{2}(\Omega)}^{2}
\end{aligned}
$$

Proof. See pages 92, 93 in [9] for proof and relation with parameters of $\left(\mathbf{P}_{w}^{\varepsilon}\right)$.

Theorem 1. Assume assumptions (A1) - (A3) hold, then there exists a unique pair $\left(\mathbf{U}^{\varepsilon}, \mathbf{V}^{\varepsilon}\right) \in H^{1}((0, T) \times \Omega)^{N} \times L^{\infty}\left((0, T), H_{0}^{1}(\Omega)\right)^{N}$ such that $\left(\mathbf{U}^{\varepsilon}, \mathbf{V}^{\varepsilon}\right)$ is a weak solution to $\left(\boldsymbol{P}_{w}^{\varepsilon}\right)$.

Proof. Use $\phi=\mathbf{V}^{\varepsilon}$ and apply Lemma 1 . Then use $\psi \in\left\{\mathbf{U}^{\varepsilon}, \partial_{t} \mathbf{U}^{\varepsilon}\right\}$. Moreover, apply a gradient to the second equation of $\left(\mathbf{P}^{\varepsilon}\right)$ and test that equation with $\nabla \mathbf{U}^{\varepsilon}$ and $\partial_{t} \nabla \mathbf{U}^{\varepsilon}$. Application of Young's inequality, use of (1) and application of Gronwall's inequality, see [2, Thm. 1], yields the existence for $\mathbf{U}^{\varepsilon}$. Then Lax-Milgram yields the existence for $\mathbf{V}^{\varepsilon}$. Uniqueness follows from the bilinearity of $\left(\mathbf{P}_{w}^{\varepsilon}\right)$. For more details, see pages 93 and 94 in [9].

\section{Upscaling the system $\left(P_{w}^{\varepsilon}\right)$ via two-scale convergence}

Based on two-scale convergence, see [1], [5], [7] for details, we obtain the following Lemma ensuring that the weak solution to problem $\left(\mathbf{P}_{w}^{\varepsilon}\right)$ has two-scale limits in the limit $\varepsilon \downarrow 0$.

Lemma 2. Assume assumptions (A0), (A1), (A2) to hold. For each $\varepsilon \in\left(0, \varepsilon_{0}\right)$, let the pair of sequences $\left(\mathbf{U}^{\varepsilon}, \mathbf{V}^{\varepsilon}\right) \in H^{1}((0, T) \times \Omega) \times L^{\infty}\left((0, T) ; H_{0}^{1}(\Omega)\right)$ be the unique weak solution to $\left(\boldsymbol{P}_{w}^{\varepsilon}\right)$. Then this sequence of weak solutions satisfies the estimate 
$\left\|\mathbf{U}^{\varepsilon}\right\|_{H^{1}((0, T) \times \Omega)^{N}}+\left\|\mathbf{V}^{\varepsilon}\right\|_{L^{\infty}\left((0, T), H_{0}^{1}(\Omega)\right)^{N}} \leq C$, for all $\varepsilon \in\left(0, \varepsilon_{0}\right)$ and there exist vector functions

$$
\begin{array}{ll}
\mathbf{u} \text { in } H^{1}((0, T) \times \Omega)^{N}, & \mathscr{U} \text { in } H^{1}\left((0, T) ; L^{2}\left(\Omega ; H_{\#}^{1}(Y) / \mathbf{R}\right)\right)^{N}, \\
\mathbf{v} \text { in } L^{\infty}\left((0, T) ; H_{0}^{1}(\Omega)\right)^{N}, & \mathscr{V} \text { in } L^{\infty}\left((0, T) \times \Omega ; H_{\#}^{1}(Y) / \mathbf{R}\right)^{N},
\end{array}
$$

and a subsequence $\varepsilon^{\prime} \subset \varepsilon$, for which the following two-scale convergences

$$
\begin{aligned}
& \mathbf{U}^{\varepsilon^{\prime}} \stackrel{2}{\longrightarrow} \mathbf{u}(t, \mathbf{x}), \quad \nabla \mathbf{U}^{\varepsilon^{\prime}} \stackrel{2}{\longrightarrow} \quad \nabla \mathbf{u}(t, \mathbf{x})+\nabla_{\mathbf{y}} \mathscr{U}(t, \mathbf{x}, \mathbf{y}), \\
& \partial_{t} \mathbf{U}^{\varepsilon^{\prime}} \stackrel{2}{\longrightarrow} \partial_{t} \mathbf{u}(t, \mathbf{x}), \quad \partial_{t} \nabla \mathbf{U}^{\varepsilon^{\prime}} \stackrel{2}{\longrightarrow} \partial_{t} \nabla \mathbf{u}(t, \mathbf{x})+\partial_{t} \nabla_{\mathbf{y}} \mathscr{U}(t, \mathbf{x}, \mathbf{y}), \\
& \mathbf{V}^{\varepsilon^{\prime}} \stackrel{2}{\longrightarrow} \mathbf{v}(t, \mathbf{x}), \quad \nabla \mathbf{V}^{\varepsilon^{\prime}} \stackrel{2}{\longrightarrow} \nabla \mathbf{v}(t, \mathbf{x})+\nabla_{\mathbf{y}} \mathscr{V}(t, \mathbf{x}, \mathbf{y})
\end{aligned}
$$

hold for a.e. $t \in(0, T)$.

Proof. See pages 95 and 96 of [9].

Using Lemma 2, we upscale $\left(\mathbf{P}_{w}^{\varepsilon}\right)$ to $\left(\mathbf{P}_{w}^{0}\right)$ via two-scale convergence.

Theorem 2. Assume the conditions of Lemma 2 are met. Then the two-scale limits $\mathbf{u} \in H^{1}((0, T) \times \Omega)^{N}, \mathscr{U} \in H^{1}\left((0, T) ; L^{2}\left(\Omega ; H_{\#}^{1}(Y) / \mathbf{R}\right)\right)^{N}$ and $\mathbf{v} \in L^{\infty}\left((0, T) ; H_{0}^{1}(\Omega)\right)^{N}$ introduced in Lemma 2 form the weak solution triple to

$$
\left(\boldsymbol{P}_{w}^{0}\right) \quad\left\{\begin{array}{l}
\int_{\Omega} \phi^{\top}\left[\overline{\mathrm{M}} \mathbf{v}-\overline{\mathbf{H}}-\overline{\mathrm{K}} \mathbf{u}-\overline{\mathrm{J}} \cdot \nabla \mathbf{u}-\frac{1}{|Y|} \int_{Y} \mathrm{~J} \cdot \nabla_{\mathbf{y}} \mathscr{U} \mathrm{d} \mathbf{y}\right] \\
\quad+(\nabla \phi)^{\top} \cdot\left(\mathrm{E}^{*} \cdot \nabla \mathbf{v}+\mathrm{D}^{*} \mathbf{v}\right) \mathrm{d} \mathbf{x}=0, \\
\int_{\Omega} \psi^{\top}\left[\partial_{t} \mathbf{u}+\mathrm{Lu}-\mathrm{G} \mathbf{v}\right] \mathrm{d} \mathbf{x}=0, \\
\int_{Y} \xi^{\top} \cdot \nabla_{\mathbf{y}}\left[\partial_{t} \mathscr{U}+\mathrm{L} \mathscr{U}-\tilde{\delta} \mathbf{v}-\tilde{\omega} \cdot \nabla \mathbf{v}\right] \mathrm{d} \mathbf{y}=0, \\
\mathbf{u}(0, \mathbf{x})=\mathbf{U}_{*}(\mathbf{x}) \quad \text { on } \Omega, \\
\nabla_{\mathbf{y}} \mathscr{U}(0, \mathbf{x}, \mathbf{y})=\mathbf{0} \quad \text { on } \Omega \times Y,
\end{array}\right.
$$

for a.e. $t \in(0, T)$, for all test-functions $\phi \in H_{0}^{1}(\Omega)^{N}, \psi \in L^{2}(\Omega)^{N}$, and $\xi \in$ $H_{\#}^{1}(Y)^{d \times N}$, where the effective coefficients $\mathrm{E}^{*}$ and $\mathrm{D}^{*}$ are given by

$$
\begin{aligned}
\mathrm{E}^{*}=\frac{1}{|Y|} \int_{Y} \mathrm{E} \cdot\left(1+\nabla_{\mathbf{y}} \mathbf{W}\right) \mathrm{d} \mathbf{y}, & \mathrm{D}^{*}=\frac{1}{|Y|} \int_{Y} \mathrm{D}+\mathrm{E} \cdot \nabla_{\mathbf{y}} \delta \mathrm{d} \mathbf{y} \\
\tilde{\delta}=\nabla_{\mathbf{y}}(\mathrm{G} \delta), & \tilde{\omega}=\nabla_{\mathbf{y}} \mathbf{W} \otimes \mathrm{G},
\end{aligned}
$$

and the tensor $\left.\delta_{\alpha \beta} \in L^{\infty}\left((0, T) \times \Omega ; H_{\#}^{1}(Y) / \mathbf{R}\right)\right)$ and vector $\mathbf{W}_{i} \in L^{\infty}((0, T) \times$ $\left.\left.\Omega ; H_{\#}^{1}(Y) / \mathbf{R}\right)\right)$ satisfy the cell problems

$$
0=\int_{Y} \Phi^{\top} \cdot\left(\nabla_{\mathbf{y}} \cdot\left[\mathrm{E} \cdot\left(1+\nabla_{\mathbf{y}} \mathbf{W}\right)\right]\right) \mathrm{d} \mathbf{y}, \quad 0=\int_{Y} \Psi^{\top}\left(\nabla_{\mathbf{y}} \cdot\left[\mathrm{D}+\mathrm{E} \cdot \nabla_{\mathbf{y}} \delta\right]\right) \mathrm{d} \mathbf{y}
$$

for all $\Phi \in C_{\#}(Y)^{d}, \Psi \in C_{\#}(Y)^{N \times N}$. 
Proof. In $\left(\mathbf{P}_{w}^{\varepsilon}\right)$, we choose $\phi=\phi^{\varepsilon}=\Phi\left(t, \mathbf{x}, \frac{\mathbf{x}}{\varepsilon}\right)$ and $\psi=\psi^{\varepsilon}=\Psi(t, \mathbf{x})+\varepsilon \varphi\left(t, \mathbf{x}, \frac{\mathbf{x}}{\varepsilon}\right)$ for the test-functions $\Phi \in L^{2}\left((0, T) ; \mathscr{D}\left(\Omega ; C_{\#}^{\infty}(Y)\right)\right)^{N}, \Psi \in L^{2}\left((0, T) ; C_{0}^{\infty}(\Omega)\right)^{N}$ and for $\varphi \in L^{2}\left((0, T) ; \mathscr{D}\left(\Omega ; C_{\#}^{\infty}(Y)\right)\right)^{N}$. Two-scale convergence limits, see [1], [5], [7], and cell-function arguments, see [6], give $\left(\mathbf{P}_{w}^{0}\right)$. Details in pages 97,98 of [9].

We have shown that upscaling system $\left(\mathbf{P}_{w}^{\varepsilon}\right)$ yields system $\left(\mathbf{P}_{w}^{0}\right)$. This system contains only PDEs with respect to $(t, \mathbf{x})$. However, an extra variable $\nabla_{\mathbf{y}} \mathscr{U}$ was needed. Removing $\nabla_{\mathbf{y}} \mathscr{U}$ needs the use of continuous semi-group theory, see papers 10 and 14 of [10], for solving the third equation of system $\left(\mathbf{P}_{w}^{0}\right)$. This leads to a non-localin-time term as a consequence of removing $\nabla_{\mathbf{y}} \mathscr{U}$.

\section{Conclusion}

Our main goal of this paper is to show that the spatial-temporal decomposition, as employed in [8], allows for the straighforward upscaling of pseudo-parabolic equations, in specific for system $\left(\mathbf{Q}^{\varepsilon}\right)$. The upscaling procedure is here performed using the concept of two-scale convergence as reported in Section 4. Moreover, the decomposition is retained in the upscaled limit. A non-local-in-time term arose when an extra variable was eliminated. The spatial-temporal decoupling showed why this non-local term is non-local in time.

In future research we intend to investigate the applicability of the spatial-temporal decomposition of our pseudo-parabolic system to perforated periodic domains, corrector estimates (convergence speed estimate) and high-contrast situations.

\section{References}

1. Allaire, G.: Homogenization and two-scale convergence. SIAM J. Math. Anal 23(6), 14821518 (1992)

2. Dragomir, S.S.: Some Gronwall Type Inequalities and Applications. RGMIA Monographs. Nova Science, New York (2003)

3. Hassanizadeh, S.M., Celia, M., Dahle, H.: Dynamica effect in the capillary pressure-saturation relationship and its impact on unsaturated flow. Vadose Zone Journal 1, 38-57 (2002)

4. Lam, T.Y.: On the diagonalization of quadratic forms. Math. Mag. 72(3), 231-235 (1999)

5. Lukkassen, D., Nguetseng, G., Wall, P.: Two-scale convergence. Int. J. of Pure and Appl. Math. 2(1), 35-62 (2002)

6. Muntean, A., Chalupecký, V.: Homogenization Method and Multiscale Modeling. No. 34 in COE Lecture Note. Institute of Mathematics for Industry, Kyushu University, Japan (2011)

7. Nguetseng, G.: A general convergence result for a functional related to the theory of homogenization. SIAM J. Math. Anal. 20(3), 608-623 (1989)

8. Peszyńska, M., Showalter, R., Yi, S.Y.: Homogenization of a pseudoparabolic system. Applicable Analysis 88(9), 1265-1282 (2009)

9. Vromans, A.J.: A Pseudoparabolic Reaction-Diffusion-Mechanics System: Modeling, Analysis and Simulation. Licentiate thesis, Karlstad University (2018)

10. Yosida, K.: Functional Analysis. No. 123 in Die Grundlehren der Mathematischen Wissenschaften in Einzeldarstellungen mit Besonderer Berücksichtigung der Anwendungsgebiete. Springer-Verlag (1965) 